which takes the maximum value of $x+y-1$ when $t=y$.

But when $B_{t}$ is placed, all remaining $B$ s fit in without further weighings. Therefore total number of weighings required $<x+y-1$.

If all the $B$ s are lighter than $A_{x}$, suppose that $A_{x+1}$ is the lightest $A$ to be heavier than $B_{y}$. As before,

$B_{1}$ moves $b_{1}-1$ places along,

$B_{2}$ moves a further $b_{2}-1$ places along,

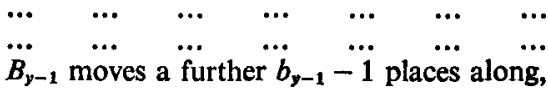

but, now

$B_{y}$ moves a further $b_{y}-1$ places along.

Therefore

$$
\begin{aligned}
z & =\left(b_{1}-1\right)+\left(b_{2}-1\right)+\cdots+\left(b_{y}-1\right) \\
& =\sum b-y,
\end{aligned}
$$

and the total number of weighings required is

$$
\begin{aligned}
\sum b & =y+z \\
& \leqslant y+x-1 .
\end{aligned}
$$

Hence in either case, $x+y-1$ weighings suffice for interlacing, and

number of weighings required in all $\leqslant U_{x}+U_{y}+x+y-1$.

Applying this to $2 n$ objects, take $x=n, y=n$; then the number of weighings required $<2 U_{n}+2 n-1=U_{2 n}$. Again, taking $x=n, y=n+1$, the number of weighings required to order $2 n+1$ objects $<U_{n}+U_{n+1}+2 n=U_{2 n+1}$. Hence the $U_{n}$ sequence gives sufficient numbers for all $n$.

Denoting the logarithmic sequence by $W_{n}$, the Cameron sequence by $C_{n}$, and the $U$ sequence by $U_{n}$, we have:

$\begin{array}{rlll}n & W_{n} & C_{n} & U_{n} \\ 8 & 16 & 16 & 16 \\ 9 & 19 & 20 & 20 \\ 10 & 22 & 24 & 23 \\ 11 & 26 & 28 & 27 \\ 12 & 29 & 32 & 31 \\ 13 & 33 & 36 & 35\end{array}$

If you continue the $C_{n}$ and $U_{n}$ sequences for $n$ up to 81 , you will observe some interesting comparisons.

Yours etc.,

STANLEY COLLINGS

The Open University, Walton Hall, Bletchley, Bucks.

\title{
A rule for turning a generalised mattress
}

(see Classroom Note 269, October 1972)

Dear Mr. Quading,

We turn our mattress much less frequently (and less regularly) than once a week, with the result that each time I have forgotten which way we turned it on the last occasion. So one day I wondered whether there were not one operation that could be repeated each time and still send the mattress to all four possible positions in turn. I am ashamed to say, since I am a group theorist by training, that it took me a finite time (even though it was 
only a few minutes) to realise that this was equivalent to asking whether the group of symmetries was cyclic, which of course it isn't.

I then saw that the problem could be solved by doing two operations alternately, which led me to make the following conjecture:

For every finite group $G$, there exists a sequence of (not necessarily distinct) elements

$a_{1}, a_{2}, \ldots, a_{d}$ such that the infinite sequence of products $p_{1}=a_{1}, p_{2}=a_{1} a_{2}, \ldots$, $p_{d}=a_{1} a_{2} \ldots a_{d}=p_{d-1} a_{d}, p_{d+1}=p_{d} a_{1}, \ldots$ (so that if $n=q d+r$, where $1<r \leqslant d$, then $p_{n}=p_{n-1} a_{r}$ ) ranges over all the elements of $G$ in succession.

Since for a cyclic group this is possible with $d=1$, the least number $d$ for which it is possible is a measure of how far $G$ deviates from being cyclic.

Having made the conjecture, I promptly forgot it until I read Note 269 , since when I have proved it (and commend it as a pretty little exercise for students in elementary group theory). It would be interesting to know if there are any interesting consequences of this result, either in the practical field (e.g., to design a machine which will pick up a symmetrical object and present it in all possible ways for some operation to be performed on each face) or possibly in the theory of permutation groups.

Yours sincerely,

University of the Witwatersrand, Jan Smuts Avenue, Johannesburg

JAMES RIDLEY

\section{Diabolic squares}

Dear SIR,

I must apologise for not having done more research before submitting Mathematical Note 3334, Diabolic Squares (October 1972). As two readers have kindly pointed out to me, diabolic squares are mentioned, for example, by Rouse Ball [1] as being synonymous with pandiagonal magic squares (of any order), which have been known for hundreds of years. The word "diabolic" to describe these was apparently first used by E. Lucas in 1882. Unfortunately I did not have my Rouse Ball with me at the time and it was a long time since I had read it, so I had forgotten this fact.

However, as I thought, Rouse Ball does not mention the property of a fourth-order pandiagonal square that if it is replicated indefinitely in each dimension, any group of four different cells forming a square array add up to the magic constant, and I confess that when I submitted my note I did not realise that all such squares possessed this property. Kraitchik [2] also does not mention this property explicitly (although it could be deduced from his Fig. 63). This I found mildly surprising as he describes in detail a similar property of fifth-order panmagic squares (as he calls them). Kraitchik also nowhere mentions the word "diabolic".

Since submitting my note, however, I have discovered that Martin Gardner [3] also mentions diabolic squares and does mention the above property of fourth-order squares. This was apparently first stated and proved in a paper by Rosser and Walker [4] in 1938.

Yours sincerely,

s. N. HIGGINS

Walton, Croft Farm Drive, Malvern, Worcs.

\section{References}

1. W. W. Rouse Ball, Mathematical recreations and essays (11th edition, revised by H. S. M. Coxeter). MacMillan (1939).

2. Maurice Kraitchik, Mathematical recreations. George Allen and Unwin (1943).

3. Martin Gardner, More mathematical puzzles and diversions. Penguin (1966).

4. Barkley Rosser and R. J. Walker, On the transformation group for diabolic magic squares of order four, Bull. Am. math. Soc. 44, 416-420 (June 1938). 